\title{
Cross-sectional study of loss of life expectancy at different ages related to firearm deaths among black and white Americans
}

\section{Bindu Kalesan, ${ }^{1,2}$ Mrithyunjay A Vyliparambil, ${ }^{3}$ Yi Zuo, ${ }^{1}$ Jeffrey J Siracuse, ${ }^{4}$ Jeffrey A Fagan, ${ }^{5}$ Charles C Branas, ${ }^{6}$ Sandro Galea ${ }^{7}$}

10.1136/bmjebm-2018-111103

${ }^{1}$ Department of Medicine, Boston University School of Medicine, Boston, MA, USA ${ }^{2}$ Department of Community Health Sciences, Boston University School of Public Health, Boston, MA, USA ${ }^{3}$ Department of Public Health, College of Health Sciences, University of Massachusetts Lowell, Lowell, Massachusetts, USA

${ }^{4}$ Department of Surgery, Boston University School of Medicine, Boston, Massachusetts, USA ${ }^{5}$ Law School and Department of Epidemiology, Columbia University, New York, New York, USA

${ }^{6}$ Department of Epidemiology, Mailman School of Public Health, Columbia University, New York, NY, USA ${ }^{7}$ Department of Epidemiology, Boston University School of Public Health, Boston, MA, USA

\section{Correspondence to:} Dr Bindu Kalesan, Department of Medicine and Community Health Sciences, Boston University School of Medicine and School of Medicine, Boston MA 02118, USA; kalesan@bu.edu

\section{Check for updates}

(-) Author(s) (or their employer(s)) 2019. No commercial re-use. See rights and permissions. Published by BMJ.

To cite: Kalesan B, Vyliparambil MA, Zuo Y, et al. BMJ Evidence-Based Medicine 2019;24:55-58.

\section{Abstract}

Understanding the life years lost by assault and suicide due to firearms among white and black Americans can help us understand the racespecific and intent-specific firearm mortality burden and inform prevention programmes. The objective was to assess national and racespecific life expectancy loss related to firearms in the USA due to assault and suicide. We used firearm mortality data available from Wideranging Online Data for Epidemiologic Research to calculate the life expectancy loss between 2000 and 2016 separately for assaults and suicides among white and black Americans. The total national life expectancy loss due to firearms was 2.48 ( 2.23 whites, 4.14 blacks) years. The total life expectancy loss in years due to firearm assault was 0.95 (0.51 whites, 3.41 blacks) and suicide was 1.43 (1.62 whites, 0.60 blacks), respectively. Firearm life expectancy loss in years at birth, 20, 40 and 60 years of age was 0.29 ( 0.22 whites, 0.56 blacks), 0.25 (0.21 whites, 0.47 blacks), 0.09 (0.10 whites, 0.08 blacks) and 0.03 (0.03 whites, 0.01 blacks) years. National firearm life expectancy loss in days from 20 to 60 years declined by 79.5 (65.8 whites, 166.3 blacks); for assault by 37.5 (18.9 whites, 141.0 blacks) and suicides by 38.7 (43.9 whites, 20.3 blacks). Americans lose substantial years of life due to firearm injury. This loss in life years is characterised by a large racial gap by age and intent. Tailored prevention programmes are needed to reduce this loss and lessen the racial gaps.

\section{Introduction}

The burden of death due to firearms is an ongoing public health problem in the USA. US men had a $21 \%$ life expectancy loss due to firearms compared with men from other countries. ${ }^{1}$ In $2016,7.8 \%$ of the years of potential life lost at 65 years was attributed to firearms. ${ }^{2}$ Although the overall age-adjusted firearm death rates remained relatively constant between 2000 and 2014, ${ }^{2}$ the death rates due to firearms varied dramatically by race in the USA during that time period. ${ }^{3}$ For at least a decade, firearm injury and death in the USA has been characterised by large race and sex disparities. ${ }^{3}$ While these disparities receive limited recognition in the popular and scientific journals, few solutions for reducing them have been developed. $^{45}$

Life expectancy is one of the most important demographic indicators that provides a measure of the expected value of future life as compared with mortality rate. Life expectancy is defined as the mean number of years a cohort of people might expect to live according to the current age-specific mortality rates. Overall life expectancy in the USA grew from 76.8 years in 2000 to 78.7 years in $2014 .^{6}$ Yet, life expectancy in the USA decreased by 0.1 from 2014 to 2015; this was the first decrease in 50 years. ${ }^{6}$ This decline continued from 2015 to 2016 with a further drop of 0.1 years. ${ }^{7}$ Current literature has analysed firearm injury deaths exclusively as per capita rates, with firearm mortality rates remaining constant from 2000 to 2014 (10.3 per 100000 on average) to a jump in 2015 (11.3 per 100 000) and 2016 (12.0 per 100 000). Very few studies have evaluated firearm injury deaths based on life expectancy or life expectancy loss. ${ }^{8}$

Given the lack of assessment of life expectancy loss related to firearms during the 21st century and the persisting high firearm mortality rate, we hypothesised that life expectancy loss is more significant among black than white Americans. Life expectancy provides a comprehensive assessment of the scope of firearm injury, providing a full accounting of the consequences of firearms. Therefore, we inform and advance the current discussion by investigating the national and race-specific life expectancy loss due to firearms in the USA. We also assessed race-specific life expectancy loss due to firearm assaults and suicides among white and black Americans at different ages.

\section{Methods}

\section{Study design}

We used national mortality data related to firearm injury during a 15-year period (2000 to 2016) from the Centers for Disease Control and Prevention (CDC) Wide-ranging Online Data for Epidemiologic Research (WONDER). ${ }^{2}$ To examine the disparities by race and intent, we obtained counts of deaths by firearm in 5-year age groups within each race and intent subgroup. We do not differentiate by sex since only 10\% of firearm deaths are women and the sample size would be inadequate 


\begin{tabular}{|c|c|c|c|c|c|c|}
\hline & \multirow{2}{*}{$\begin{array}{l}\text { Rate } \\
\text { Per } 100000\end{array}$} & \multicolumn{5}{|c|}{ Life expectancy loss, years } \\
\hline & & Total & Birth & 20 & 40 & 60 \\
\hline \multicolumn{7}{|l|}{ All } \\
\hline All & 10.5 & 2.48 & 0.29 & 0.25 & 0.09 & 0.03 \\
\hline Assault/legal & 3.9 & 0.95 & 0.13 & 0.11 & 0.02 & 0.003 \\
\hline Suicide & 6.2 & 1.43 & 0.14 & 0.13 & 0.07 & 0.03 \\
\hline Unintentional & 0.2 & 0.05 & 0.01 & 0.004 & 0.002 & 0.0005 \\
\hline Undetermined & 0.1 & 0.02 & 0.002 & 0.002 & 0.001 & 0.0002 \\
\hline \multicolumn{7}{|l|}{ White } \\
\hline All & 9.6 & 2.23 & 0.22 & 0.21 & 0.10 & 0.03 \\
\hline Assault/legal & 2.1 & 0.51 & 0.07 & 0.05 & 0.01 & 0.002 \\
\hline Suicide & 7.2 & 1.62 & 0.16 & 0.15 & 0.08 & 0.03 \\
\hline Unintentional & 0.2 & 0.05 & 0.01 & 0.004 & 0.002 & 0.0005 \\
\hline Undetermined & 0.1 & 0.02 & 0.002 & 0.002 & 0.001 & 0.0002 \\
\hline \multicolumn{7}{|l|}{ Black } \\
\hline All & 19.3 & 4.14 & 0.56 & 0.47 & 0.08 & 0.01 \\
\hline Assault/legal & 16.0 & 3.41 & 0.47 & 0.39 & 0.05 & 0.01 \\
\hline Suicide & 2.6 & 0.60 & 0.07 & 0.06 & 0.02 & 0.01 \\
\hline Unintentional & 0.3 & 0.06 & 0.01 & 0.01 & 0.001 & 0.0003 \\
\hline Undetermined & 0.1 & 0.02 & 0.003 & 0.002 & 0.0004 & 0.0001 \\
\hline
\end{tabular}

The loss of life expectancy in years after removing the disease-specific deaths from all-cause deaths.

for calculation of life expectancy loss. The population counts were also obtained from CDC-WONDER for national and subgroups of race, while for intent we used the national estimates. ${ }^{2}$

\section{Statistical analysis}

Age-adjusted rates were calculated using national population estimates from 2000 in the 19 age groups, each of which was in 5-year intervals. ${ }^{9}$ These age groups are commonly used in the calculation of life expectancy. We calculated national and race-specific life expectancy in 19 age groups for all-cause deaths ( $\mathrm{LE}_{\mathrm{a}}$ ) using period life tables $^{10}$ and then subtracting type of death from all-cause deaths to calculate life expectancy ( $\mathrm{LE}_{\mathrm{a}-\mathrm{f}}$ ) for each age group. The loss in life expectancy due to firearm suicide and assaultive deaths for each age group in years and days was also calculated $\left(\mathrm{LE}_{\mathrm{f}}=\mathrm{LE}_{\mathrm{a}-\mathrm{f}}-\mathrm{LE}_{\mathrm{a}}\right)$. The life expectancy loss between ages 20 and 60 was calculated as the difference in life expectancy at age 20 and 60 . R statistical software was used for analysis. ${ }^{11}$ The program used in calculation of life expectancy can be downloaded here.

\section{Results}

Comparison of life expectancy loss for these age groups and by race is shown in table 1 . The total, at birth, 20, 40 and 60 years of age for national firearm life expectancy loss was 2.48 (2.23 whites, 4.14 blacks), 0.29 (0.22 whites, 0.56 blacks), 0.25 (0.21 whites, 0.47 blacks), 0.09 (0.10 whites, 0.08 blacks) and 0.03 (0.03 whites, 0.01 blacks) years (table 1). The total life expectancy loss in years for firearm assault was 0.95 ( 0.51 whites, 3.41 blacks) and suicide was 1.43 ( 1.62 whites, 0.60 blacks), respectively.

The magnitude of life expectancy loss in days due to firearms at each age group among national and white and black Americans is shown in figure 1 . Nationally, firearm life expectancy loss from 20 to 60 years of age demonstrated a drop of 79.5 (90.2 to 10.7) days. This life expectancy loss drop in the same age period was 65.8 (77.6 to 11.8 ) in whites and 166.3 (171.1 to 4.8) days in blacks. The drop in national firearm assault life expectancy loss in days was 37.5 (38.5 to 1.0 ); among whites, it was 18.9 (19.7 to 0.8) and blacks it was 141.0 (143.2 to 2.2 ) days during the same age period. In the same age period, the drop in national suicide firearm life expectancy loss was 38.7 days (48.1 to 9.4); among whites, it was 43.9 (54.5 to 10.6), and among blacks, it was 20.3 (22.8 to 2.5).

\section{Discussion}

We found substantial life expectancy loss to gun violence in the USA since the turn of the 21st century. Life expectancy loss usually slowly declines with age. We observed a sudden drop at age 20 among black Americans for assaults and white Americans for suicides. Life expectancy loss below age 20 is primarily due to assault mortality among black Americans and that past the age of 20 is driven primarily by suicide deaths among white Americans. The overall life expectancy loss is twice as high among blacks compared with whites and is driven by substantially higher homicide rates among blacks up to age 20 . The life expectancy loss due to suicide among whites over age 20 is greater than blacks (10.4 vs 2.5 days at age 60 ), and this does not offset the overall higher firearm life expectancy loss among blacks compared with whites. This divergent race-specific life expectancy loss by age is in line with current evidence that suggests vast race differences in the years of life lost due to firearms before age 65 years. ${ }^{12}$ Our finding that the life expectancy loss related to suicides among whites does not offset the loss among blacks is indicative of persisting disparities in homicide among younger age groups.

To our knowledge, this is the only contemporary report quantifying the magnitude of life expectancy loss at different age groups due to assault and suicide firearm mortality among black and white Americans. A similar assessment of life expectancy loss using firearm mortality data (28663 firearm deaths) in the year 2000 reported that reduction in life expectancy due to firearms was 103.6 days, and racially disparate with a 150.7-day loss for white men and 361.5 days for black men. ${ }^{13}$ Our study using cumulative data from 2000 to 2016 demonstrates a total firearm life expectancy loss of 905.2 days (2.48 days), which is nine times greater than observed in 2000, indicating increasing life expectancy loss by year. Additionally, the previously reported twice greater loss among blacks as compared with whites in $2000^{13} 14$ 

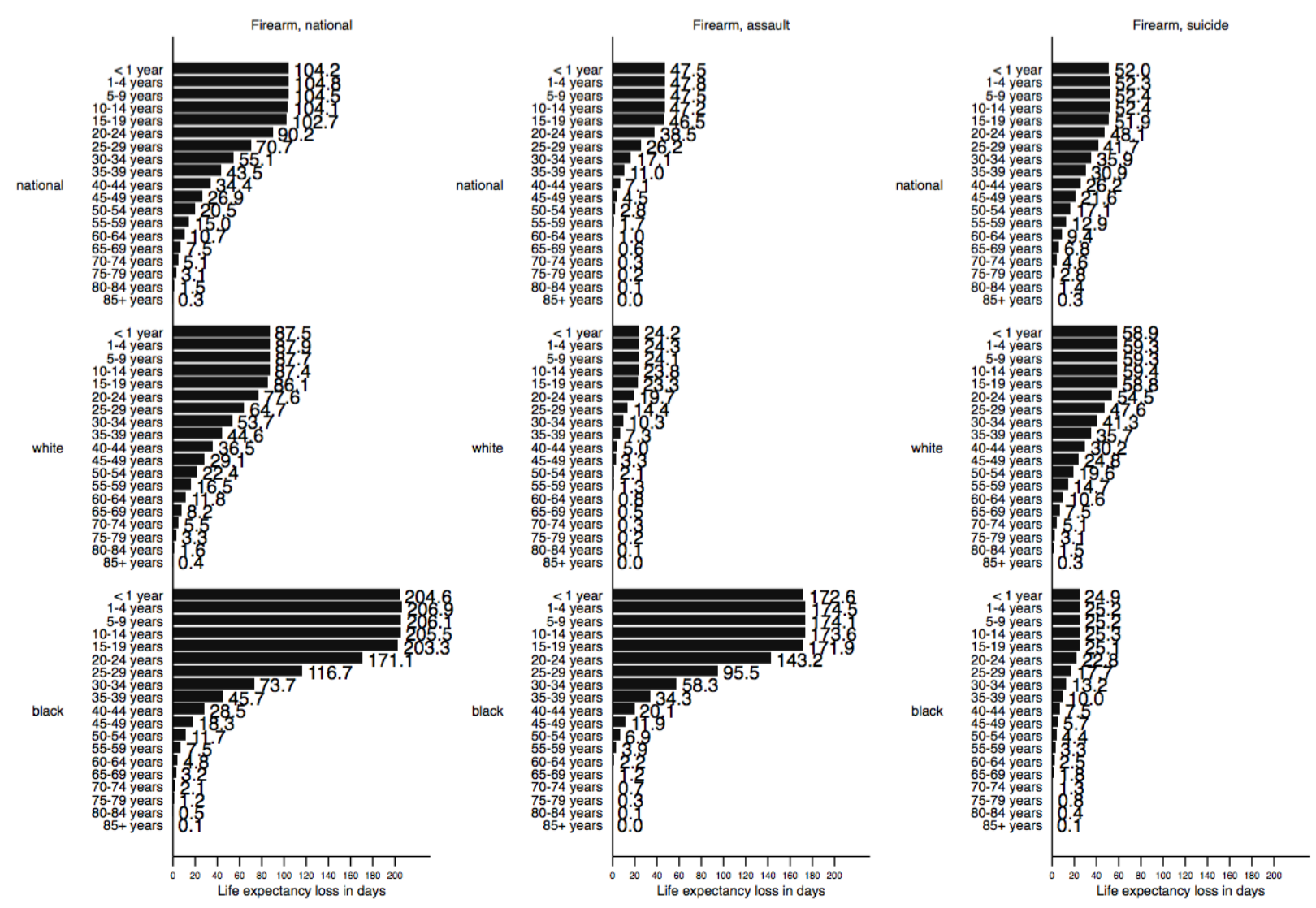

Figure 1 National, intent-specific and race-specific life expectancy loss in days related to firearms at 5-year age groups, 2000-2016. All values are in days.

also is reflected in our study, but at a much greater magnitude in the respective racial subgroups (4.14 years among blacks and 2.23 years among whites).

The expected pattern of life expectancy loss attributed to deaths from all causes is a gradual decline with increasing age, indicating that fewer years are lost in an older population, similar to that of life expectancy for each age period. ${ }^{10}$ Therefore, the resultant life expectancy loss at 20 years is likely to be followed by a steep drop in life expectancy loss to older age groups indicating that most of the life expectancy loss due to firearms occurs among younger people $<20$ years rather than among older people. Our study demonstrates a sudden drop in life expectancy due to a considerable life expectancy loss from 20 to 60 years that was 2.5 times greater among blacks than whites. This disparity indicates that black Americans were shot and killed when they were younger much more than white Americans. Among black Americans, a large life expectancy drop was observed from 20 to 60 years of age, indicating that the majority of life expectancy loss occurs among young black Americans. Assaultive injuries among black Americans drove this premature mortality due to firearms. On the other hand, among white Americans, the drop is relatively minor for overall and assault firearm deaths. Instead, the drop in life expectancy loss related to suicide firearm mortality for white Americans is more substantial than for black Americans, indicating that suicide mortality disproportionately affects older white Americans.

This study was limited by non-standardised and potentially inaccurate reporting of Hispanic ethnicity from the year 2000, and that prevented investigation of firearm death by different ethnic groups. Future work may fruitfully use more accurate data that can allow these estimates for differentiation between Hispanics and non-Hispanics. This limitation is shared by the majority of crime and firearm data that does not uniformly report data by ethnicity across time and also due to the lack of reliable estimates for the Hispanic population. Additionally, black Americans may include several ethnicities, which are not taken into consideration due to the lack of details regarding ethnicity in the data.

To summarise, from 2000 to 2016, life expectancy loss due to firearms among Americans was high and exhibited distinct racial differences and across age and intent. The racial disparities in firearm-related life expectancy loss demonstrated in 2000 remained during the following 17 years. In the absence of comprehensive firearm legislation, targeted prevention programmes and policies are needed to mitigate the racial firearm injury gaps in the USA.

Contributors BK and SG conceived the study. YZ and BK obtained data. MAV, YZ and BK performed data analysis. All authors wrote and finalised the manuscript.

Funding National Institute of Justice, Department of Justice, 2017-IJ-CX-0018.

Competing interests None declared.

Patient consent Not required.

Provenance and peer review Not commissioned; externally peer reviewed. 
Data sharing statement All data used in the study are available online and can be downloaded from the CDC website (https:// www.cdc.gov/injury/wisqars/fatal.html).

Author note The data used are secondary data.

\section{References}

1. Fenelon A, Chen LH, Baker SP. Major causes of injury death and the life expectancy gap between the united states and other high-income countries. JAMA 2016;315:609-11.

2. Centers for Disease Control and Prevention. Multiple Cause of Death 1999-2016, CDC WONDER Online Database. Data are from the Multiple Cause of Death Files, 1999-2016, as compiled from data provided by the 57 vital statistics jurisdictions through the Vital Statistics Cooperative Program. http://wonder.cdc.gov/mcd-icd10.html (Accessed 13 Mar 2018).

3. Kalesan B, Adhikarla C, Pressley JC, et al. The hidden epidemic of firearm injury: increasing firearm injury rates during 2001-2013. Am J Epidemiol 2017;185:546-53.

4. Keppel KG. Ten largest racial and ethnic health disparities in the United States based on Healthy People 2010 Objectives. Am J Epidemiol 2007;166:97-103.

5. Riddell CA, Harper S, Cerdá M, et al. Comparison of rates of firearm and nonfirearm homicide and suicide in black and white non-hispanic men, by U.S. state. Ann Intern Med 2018;168:712-20.
6. Xu J, Kochanek KD, Murphy SL, et al. Mortality in the United States, 2012. NCHS Data Brief 2014(168):1-8.

7. Kochanek KD, Murphy S, Xu J, et al. Mortality in the United States, 2016. NCHS data brief 2017;293:1-8.

8. Fowler KA, Dahlberg LL, Haileyesus T, et al. Firearm injuries in the United States. Prev Med 2015;79:5-14.

9. Inskip H, Beral V, Fraser P, et al. Methods for age-adjustment of rates. Stat Med 1983;2:455-66.

10. Molla MT, Wagener DK, Madans JH. Summary measures of population health: methods for calculating healthy life expectancy. Healthy People Statistical Notes, no. 21. Hyattsville, Maryland: National Center for Health Statistics, 2001.

11. R Development Core Team (2010), 2010. R: A language and environment for statistical computing. R Foundation for Statistical Computing, Vienna, Austria. ISBN 3-900051-07-0 www.R-project.org/

12. Centers for Disease Control and Prevention (CDC). Firearm-related years of potential life lost before age 65 years-United States, 1980-1991. MMWR Morb Mortal Wkly Rep 1994;43:609-11.

13. Lemaire $\mathbf{J}$. The effect of firearm deaths on life expectancy and insurance premiums in the United States. LDI Issue Brief 2005;11:1-4.

14. Lemaire J. The cost of firearm deaths in the United States: reduced life expectancies and increased insurance costs. Journal of Risk Insurance 2005;72:359-74. 